\title{
A CONCEPTUAL MODEL TO FRAME GOALS OF SUSTAINABLE DEVELOPMENT
}

\author{
C. BOGLIOTTI ${ }^{1} \&$ J.H. SPANGENBERG ${ }^{2}$ \\ ${ }^{1}$ International Center for Advanced Agronomic Studies in the Mediterranean, Mediterranean Agronomic Institute, Bari, Italy. \\ ${ }^{2}$ Sustainable Europe Research Institute, Bad Oeynhausen, Germany.
}

\section{ABSTRACT}

The exclusive concept and criteria of sustainability does not seem enough to cope with the need and the complexity of our modern society, which seeks a balance between material and non-material values. A conceptual model is presented for the development and evaluation of goals for sustainable development by combining explicit notions of governance and ethics in the complex field of sustainability. Thus, the paper is aimed at exploring the interlinkages established among sustainability, governance and ethical variables. The chaotic arena of these variables - although dependent on each other - is systematised in distinct and discrete fields. The framework represents a back-to-basis approach, transferable to any condition, in which the functions are: (1) sustainability (S), a field of macro-variables underlying normative targets and criteria to enhance social, economic and environmental sustainability; (2) governance $(\mathrm{G})$, a field of macro-variables pertinent to the organisation of the civil society and influencing the organisation of the social system to ensure sustainability; (3) ethics (€), a field of principles used to explicitly orient goals and targets of sustainable development towards a higher level of values that clearly determine the future paths of sustainability. The outcome of the methodology consists of a matrix that translates some of the $\mathrm{S}-\mathrm{G}-€$ interlinkages into multidimensional criteria to be used for framing sustainable development into specific multidimensional goals for project or strategy development. An application of the theoretical model is given in the last part of this paper.

Keywords: ethics, governance, sustainability, sustainable development, water resources.

\section{INTRODUCTION}

Since the publication of the Brundtland Report [1], sustainable development has become a major issue for institutions, stakeholders, decision makers, designers or evaluators of projects, programmes and strategic plans in developed and developing countries. Local, national and international institutions and stakeholders deal with more and more questions on: (1) How do we orient towards sustainable development goals that may be embedded in future projects or strategic plans? (2) What are the relevant criteria and variables to be considered when developing goals for sustainable development? (3) Are design and evaluation criteria, contained in currently used guidelines, aimed at explicitly orienting public discourses or programmes and projects towards sustainable development? The subjective approach to sustainable development implies a certain complexity in deciding the scheme that is better oriented towards sustainability. Orienting goals towards sustainable development requires a methodology to develop well-framed schemes across a large diversity of criteria and variables, which are often combined in a fuzzy way. The problem is that many methods used today to assess or plan sustainable development are like black boxes containing an undifferentiated number of explicit and implicit variables of sustainability or governance or ethics. Their interlinkages are not always explicitly and analytically considered in project design and assessment, reducing comprehensiveness and integration of the approach. But is governance an implicit subfunction of sustainability? Do we have to treat the notions of governance, although complementary to sustainability, as a separate field? The general paradigms, framing relationships of sustainability vs. governance, are mainly centred on the fact that sustainability must be adequately plunged in a system of good governance [2]. Also, ethics is essential to determine the quality of choices for sustainable development [3]. But what are the ethical principles inspiring sustainability? How do we establish useful interlinkages between sustainability and ethics? The problem is that the ethical principles are generally hidden in the sustainability design 
and evaluation process. They should rather be explicitly expressed and systematised, possibly in a separate field, and used as a reference point for determining the orientation of sustainable development. Ethics is attracted by the concepts of sustainability and governance, but its combination with the notions of these two fields deserves further exploration. Their separate use expresses conditions to enhance the development of separate subsystems and does not really enhance integration and comprehensiveness in designing and assessing sustainable development.

\section{DRAWING THE MODEL'S INSPIRATION FROM THE EUROPEAN UNION PROCESS}

The purpose of this paper is to present a comprehensive method, inspired by a new holistic and transferable approach for the evaluation or determination of sustainable goals on the basis of integration of notions of sustainability, governance and ethics. The method is not geared to specific cases on the basis of their sustainability. It is rather a more general underlying criterion for the integration of the essence of ethics and governance in sustainability, inspired by the existing literature [2-6], but not analytically explored with satisfactory results. Furthermore, the proposed approach is inspired by the EU values prevailing in the Treaty of Amsterdam, Article 2, further developed in the published European Commission Communication 'Guidelines for Programmes in the period 2000-2006 (OJ 1999/C 267/02)' and in the acts of the Lisbon Process [7]. But perhaps the most relevant EU document for the contextualisation of ethics and governance in designing and assessing sustainable development is the Framework Programme (FP) for Research, Technology and Development (RTD) [8]. The texts of the FP explicitly link the design and assessment of project objectives or goals to the EU aim of integrating notions of governance and ethics into sustainable development. The FP explicitly intends to enhance paths of sustainable development that go further beyond reductionism and compartmentalised concepts, encompassing the integration of sustainability, governance and ethical variables. Unfortunately, there are no guidelines given today to systematise the integration of these compartmentalised concepts.

\section{METHODOLOGICAL PRINCIPLES}

The model represents a back-to-basis heuristic approach, at the base of which are three fields of macro-variables: (1) sustainability (S), a field of macro-variables defining theoretical and normative criteria for sustainable social, environmental and economic development; (2) governance (G), a field of macro-variables pertinent to the organisation of the society, influencing the social organisation and ensuring the realisation of sustainability; (3) ethics $(€)$, a field of macro-variables inspired by the values residing in the motivation of individuals to determine the orientation of choices and paths of sustainability and governance. In this paper, a macro-variable is a combination of subsector (e.g. economic or socioeconomic) measurable variables (e.g. income, economic growth, production, productivity and so on). As we will see later in this paper, the macro-variable embeds the concepts imperative for sustainability and governance. The multidimensional interlinkage of S, G and $€$ inscribe the space in which early-stage project design and assessment should be conceptually inscribed (Fig. 1).

The triangle in Fig. 1 represents the conceptualisation at the base of the model, where the space delimited by the simultaneous interaction of $S, G$ and $€$ is explored. The model attempts, first, the systematisation of the number of variables often randomly encompassed in the current concepts and methods used to design and assess sustainable development. We tried to differentiate them into discrete groups of macro-variables in each S, G and $€$ field. Although the attribution of macro-variables to one or the other field remains a subjective exercise, care has been taken to explicitly characterise $S$, $\mathrm{G}$ and $€$ fields with macro-variables that are relevant to the characterisation of a given field.

It is important to underline that the presented methodological outline intends to stimulate a discussion about the reliability of a method based on separate, pseudo-orthogonal sustainability, governance and ethic fields, the potential and the limits to this regard and the need of more analytical forms to 


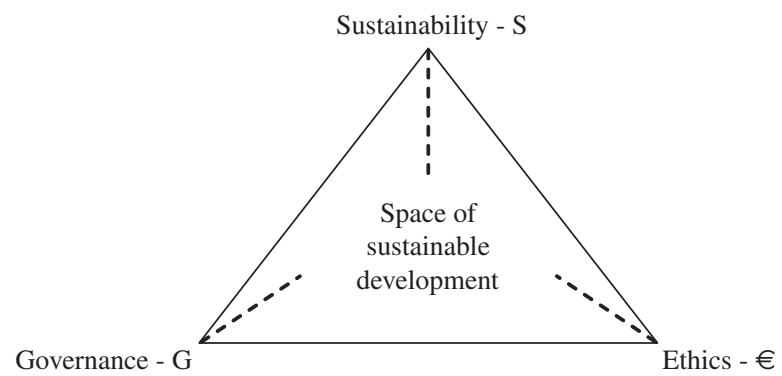

Figure 1: The S-G-€ triangle and the space of project design and assessment.

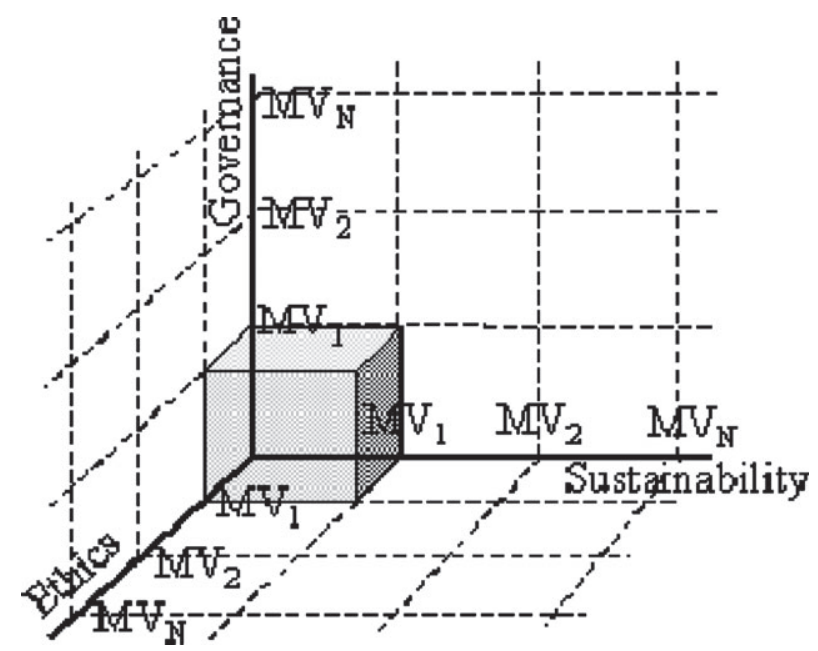

Figure 2: 3D matrix representating the multidimensional interlinkages among $S, G$ and $€$, where $\mathrm{MV}=$ macro-variable. Each interlinkage is graphically represented by a cubic form.

frame sustainable development in a process of goal deliberation or schematisation. However, sustainability, ethics and governance are still traditionally regarded as interdependent fields, and their functional organisation in an orthogonal system, although greatly desired by the scientific community, planners and evaluators, is still considered unjustifiable. We have decided to use a heuristic device based on a 3D matrix for the representation of the $S-G-€$ field. The matrix constitutes the graphical and analytical tool to explore the complex nature of the $\mathrm{S}-\mathrm{G}-€$ field and the internal interlinkages (Fig. 2).

\subsection{Sustainability $(\mathrm{S})$}

\section{THE THREE FUNCTIONS OF SUSTAINABLE DEVELOPMENT}

The concept of sustainability and the related sustainability criteria and imperatives in social, economic, environmental and institutional dimensions have been extensively described in the literature by Spangenberg et al. [9, 10], Serageldin [11], Funtowicz [12] and Rosner [13]. Synthesising from the above literature, concerns of environmental and social dimensions address respectively (1) all bio-geological processes and elements involved and (2) intra-individual capacities and culture. 
Table 1: The macro-variables that characterise the three dimensions of sustainability (modified after Valentin and Spangenberg [14]).

\begin{tabular}{ll}
\hline Dimension of sustainability & Macro-variable $(\mathrm{MV})$ \\
\hline Economic & Competitiveness $\left(\mathrm{MV}_{1}\right)$ \\
Social & Cohesion $\left(\mathrm{MV}_{2}\right)$ \\
Environment & Limit throughput $\left(\mathrm{MV}_{3}\right)$ \\
\hline
\end{tabular}

The criteria of economic dimension or man-made capital address all forms of economic activities, rules, actors and capacities.

The institutional dimension is considered by some authors to be a dimension of the sustainability field and supposed to address institution and participation of the society in decision-making $[9,10]$. It might be too reductive to use the traditional concept of institutional dimension to embed the concept of governance in the sustainability function. As we will see later in the next section of this paper, the concept of governance encompasses the institutional dimension in a systemic vision, enhancing the degree of good interdependences of empowered institutions, groups, communities and citizens to ensure sustainable development. Thus, we have assumed the institutional dimension to pertain to the sphere of governance and not to the field of sustainability. In the end, we consider the field of sustainability as a vector resulting from a combination of normative macro-variables generalised from the societal, environmental and economic dimensions and their interlinkages. The discreteness proposed for the field of sustainability is given by a set of macro-variables generalised from the imperatives of Valentin and Spangenberg [14] for the social, economic and environmental dimensions, and being respectively, safeguard cohesion, improve competitiveness and limit throughput. Linking these imperatives to the three dimensions of sustainability is nothing but the application of the Kantian 'Categorical Imperatives' to lifestyle and environmental issues [14, 15]. These imperatives can indeed be treated as macro-variables of sustainability, as they are described in the literature not only as imperatives or criteria but also as dynamic variables having an influence on sustainability $[11,12,14]$. These were the main sustainability targets in Agenda 21 [16] and in the Summit of Johannesburg. Then, we assume the S-field to be encompassed by three main macro-variables - 'cohesion', 'limit throughput' and 'competitiveness' - characterising respectively the social, environmental and economic dimensions (Table 1). However, according also to Valentin and Spangenberg [14], it would be reductive to link the concept of space of sustainability to the fulfilment of criteria related to the single macro-variables of Table 1 . This concept should be extended also to the space delimited by the integration or interlinkages of these variables. Thus, we represent the concept of space of sustainability with the graphical function of Fig. 3. An extensive description of the meaning of the six macro-variables in the triangle in Fig. 3, used for the discreteness of the S-field, is described in detail by Valentin and Spangenberg [14].

\subsection{Governance $(\mathrm{G})$}

Governance is that state of minimum regime based on established rules, collective capabilities and knowledge, integration of societal parties or components, roles, participation and interdependences among individuals, households, groups and institutions. Such a type of regime is at the base of governance and enhances sustainability through the tool of policy. The institutional dimension, although important, does not constitute a main variable of governance, as the concept of governance is centred 


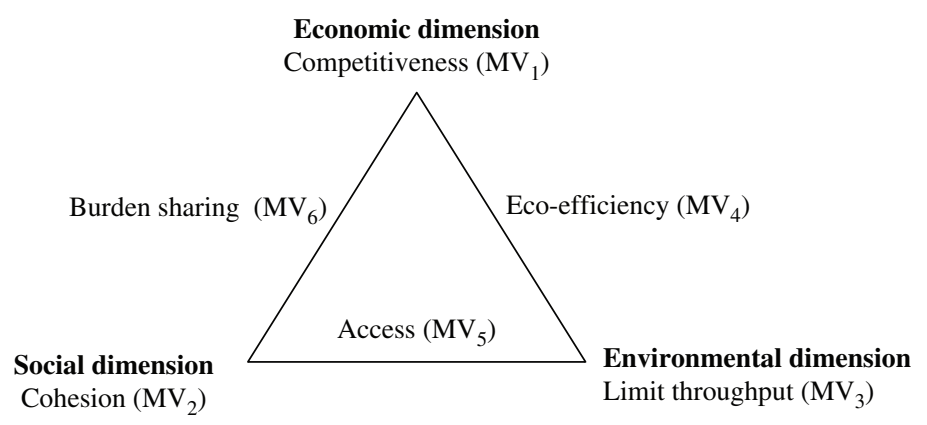

Figure 3: The triangle of sustainability is used as a graphical function to represent the conceptual space that embeds sustainability. The new macro-variables $\mathrm{MV}_{4}, \mathrm{MV}_{5}$ and $\mathrm{MV}_{6}$ help in the interpretation of the interlinkages among $\mathrm{MV}_{1}, \mathrm{MV}_{2}, \mathrm{MV}_{3}$

on the collective capacity to govern without a government [17]. Governance as such should rather be characterised by the combination of macro-variables describing the dynamics of societal participation, knowledge society and societal capability, as these elements form the basis for building a system of societal interdependences.

\subsubsection{The triangle of governance}

Although we recognise the high complexity, we assume that in a simplified, but justifiable, fashion, governance can be described as the space embedded by three main macro-variables: (1) knowledge; (2) critical mass and (3) capacity. The selection of the variables is justified by the fact that the 'trio' composed of knowledge, critical mass and capacity can well operationalise the main dimensions of governance stated in the White Book on Governance [18], where high societal participation, diversity of stakes and cultures in networking, societal knowledge to bridge different cultures and positions, and system capability are the main pillars. The proposed variables encompass and simplify the multidimensional concerns of governance. As we will see later, the bilateral or bidimensional interlink between knowledge, critical mass and capacity can be conceptually operationalised by interlinkages, each of them being expressed by a macro-variable.

- Knowledge (KN)-Agood governance depends on the variable 'knowledge', shared by the different components of society, from individuals to groups and institutions. Knowledge is an important element influencing the orientation and mode of governance. Building shared, factual knowledge is at the base of the knowledge society, necessary - although not necessarily sufficient - for bridging the positions and interests of different stakeholders, institutions, citizens and societal groups, and enabling self-organisation for effective governance. The knowledge based on transdisciplinarity helps to build comprehensive awareness, conveying the diversity of expertises, from technical to economic, social and humanistic sciences, to tackle problem resolution with a holistic approach.

- Critical mass $(C M)$ - This macro-variable is proposed since the system of governance builds on the rate of participation, co-decision or more simply - involvement of different people and stakes in managing and implementing the system itself or actions relevant to ensuring sustainability. Critical mass includes two concepts: (1) the integration of the diversity of notions, established by the overall volume of integrated disciplines and knowledge and (2) the integration of people, established by the co-operation of a high diversity of institutions, stakeholders and citizens [2, 17]. If critical mass is low, there is no strong condition for sustainability. 
- Capacity $(C A)$ - This macro-variable is related to concepts such as expertise, ability and readiness of the system to ensure sustainable development. Capacity is a 'dominium' in which the entities involved can act in certain ways and have the assets and/or power to accomplish objectives for progress [19]. Capacity is a key element to improve governance. Its design and evaluation are difficult, as progress varies greatly depending on the specific reference values of the evaluator. However, there is a general acceptance that the greater the collective capacity in the society, the higher the opportunity of governance. Effective capacity depends on the quality of collaboration, communication, reciprocal understanding and participation of people and institutions in the process of governance.

- $\mathrm{KN}-\mathrm{CM}-\mathrm{CA}$ interlinkages - The combination of $\mathrm{KN}-\mathrm{CM}-\mathrm{CA}$ variables is possible and varies with the different levels at which governance and sustainability are desired: (1) homogeneous contexts of local households or communities; (2) homogeneous contexts of catchment to watershed boundaries; (3) homogeneous contexts of transwatershed or subnational scale; (4) homogeneous contexts of international or transnational space with higher and more complex levels of interdependences. $\mathrm{KN}, \mathrm{CM}$ and $\mathrm{CA}$ variables express the necessary conditions of governance without giving information on the character of their interlinkages. The connections among KN, CM and $\mathrm{CA}$ are encompassed in the triangle in Fig. 4. The interlinkages in Fig. 4 establish the operability criteria for a balanced integration of $\mathrm{KN}, \mathrm{CM}$ and $\mathrm{CA}$. The triangle of governance illustrates the following interlinkages: (1) the expression of diversity in communication in society, (2) the collective preventive planning and (3) the diversity of planning and management. Balancing KN and $\mathrm{CM}$ establishes an interlinkage based on the involvement of high diversity and equity of access to knowledge through the enforcement of networks of a high critical mass of stakeholders, citizens and institutions, including genders and minorities. The second interlinkage between KN and CA prompts collective capacity to make the best use of shared factual knowledge to develop preventive planning to mitigate the effects of system perturbations. The third interlinkage trades $\mathrm{CM}$ and $\mathrm{CA}$ for strengthening the bulk of societal participation in planning and management. Unfolding the triangle of governance and interlinking the $\mathrm{KN}, \mathrm{CM}$ and $\mathrm{CA}$ variables with the macro-variables of sustainability enable the exploration of the interlinkages between S and $\mathrm{G}$. The choice of accounting only the three main variables of the G-field is enforced by the need for prioritising and reducing the number of interlinkages to be explored, while at the same time leaving the choice to extend, in the future, the exploration of interlinkages involving all the variables of the triangle of governance (Fig. 4).

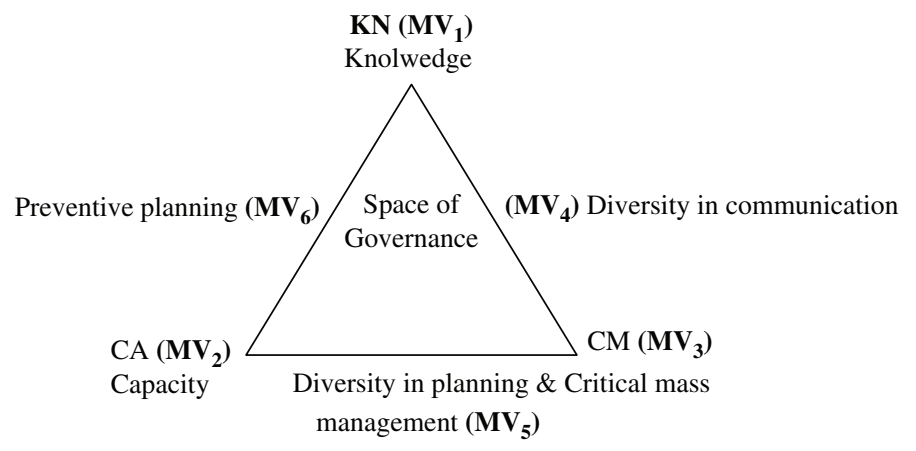

Figure 4: Graphical representation of the space of governance. $\mathrm{MV}_{1-3}$ are the macro-variables and the corners of the triangle are the main macro-variables $\left(\mathrm{MV}_{n}\right)$. 
Table 2: Ethical principles proposed as macro-variables (MV) of ethics.

\begin{tabular}{ll}
\hline Macro-variables & Characterisation \\
\hline Prosperity $\left(\mathrm{MV}_{1}\right)$ & System development in material and non-material happiness \\
Fraternity $\left(\mathrm{MV}_{2}\right)$ & Pursue the 'me and you' \\
Peace $\left(\mathrm{MV}_{3}\right)$ & Non-conflicting co-existence of different desires and stakes \\
Wisdom $\left(\mathrm{MV}_{4}\right)$ & Enhancement of conflicts, crisis and degradation \\
Truth $\left(\mathrm{MV}_{5}\right)$ & Trustfulness in relationships \\
Ecological responsibility $\left(\mathrm{MV}_{6}\right)$ & Collective responsibility to respect and restore biodiversities \\
Ecological enrichment $\left(\mathrm{MV}_{7}\right)$ & Minimal use of resources \\
Individual life $\left(\mathrm{MV}_{8}\right)$ & Rights of individuals to material and non-material happiness \\
Living in community $\left(\mathrm{MV}_{9}\right)$ & Solidarity in citizenship \\
\hline
\end{tabular}

\subsection{Ethics $(€)$}

Ethics is conceptually incorporated in the framework by means of principles that can positively support sustainability. The sphere of ethics is constellated by different types of ethical values. We excluded the ones based on ego satisfaction, consumerism or 'me or you' business as usual, as these kinds of ethical principles do not support sustainable development as such. Ethical principles should be sought to support the orientation towards sustainable development, accounting for more reflexive and modern economic, social and ecological models [20]. The kind of ethics referred to in this paper encompasses the main motivations for sustainability that reside as non-expressed value in the individuals. The use of ethics in the model helps to express more explicitly those values, which are attracted by sustainability but remain often hidden. The relevant point of the discussion is not where we stand with a certain option for sustainability or when exactly we should change from one option to the other, but in what direction we move and what kind of ethical motivations move us towards certain choices. If ethics is an important device to guide and assess sustainable development, it must be explicitly and analytically linked to notions, models or variables of sustainability [20, 21]. Framing sustainability in project design and assessment must go beyond the concept of development explained with the exclusivity of sustainability $[6,22])$. To give a practical example, nine ethical principles are proposed as macro-variables of the $€$ field to support an 'ethical' orientation of sustainable development. Although the ethical principles of Table 2 pervade the whole sphere of sustainability, some principles are more pertinent than others when considering the interlinkage with one or the other dimension of sustainability.

\section{THE SPACE OF INTERACTION AMONG SUSTAINABILITY (S), GOVERNANCE (G) AND ETHICS (€)}

The systematisation of $S, G$ and $€$ in different groups of variables, although a subjective exercise, opens further discussion on the type of variables to be arranged in each field. However, it gives an opportunity to develop an early project design and assessment, accounting for a more comprehensive concept of sustainable development, extended along a multidimensional system of governance and ethical variables. It would be too reductive and not the only scope of this paper to frame goals of sustainable development accounting for separate, although explicitly grouped, macro-variables of sustainability, governance and ethics. Separate S, G and € fields only express a few partial, although necessary, conditions to frame sustainable development in early-stage project design, keeping alive 
the separate three functions and without giving any explicit information on the overall volume of the S-G- $€$ field (the cube represented by the 3D matrix in Fig. 2) delimited by the interlinkages. It is very important to pay attention to the interlinkages, as they constitute a tool for establishing a balanced integration of $\mathrm{S}, \mathrm{G}$ and $€$ in project development. Exploring the meaning of the interlinkages should also be a target of future research, to further the knowledge of the nature of the S-G-€ field. The theoretical part of the method may also be of interest to future mathematical modelling development. However, the operability of the method is centred on the translation of the theoretical simultaneous interaction of macro-variables of the $\mathrm{S}-\mathrm{G}-€$ framework into a scheme of headings of interlinkages, usable as a preliminary guide for planners and evaluators. The simultaneous interaction of $\mathrm{S}, \mathrm{G}$ and $€$ variables is framed by the theoretical 3D matrix in Fig. 2, where the $S-G-€$ interlinkages (ITKG), due to the connection between all the $S$ and $G$ macro-variables at selected $€$ variables (the ethical principles), are inscribed.

\subsection{An outline of the multidimensional interlinkages}

The most critical part of the model is the determination of the matrix of Fig. 2, which is based on the most plausible interpretation of the $S, G$ and $€$ interlinkages. Interpretation of the interlinkages requires a conceptual balanced accountability among $S, G$ and $€$. The result consists in the definition of headings that establish the generic operability of the interlinkage among $\mathrm{S}, \mathrm{G}$ and ethical principles (Table 3). The interlinkages have been explored and formulated by unfolding the concept of multidimension integration at specific ethical principles. In more practical terms, they are the results of the attempt made to answer the question on how to develop governance at each sustainability level in regard to the ethical principles of Table 2. Although the interpretation of the multidimensional interlinkages may seem to be arbitrary, the process is inspired by implicit criteria of sustainable development consolidated in the existing literature: from reflexivity, collective competitiveness and diversity of culture in decision making to the integration of concepts like 'material and non-material', ethical manipulation of individual, ecocentrism, equity, union of different cultures, trust or transparency and spiritual benefits in economic, social and environmental sustainability [3, 6, 20-22]. The interpretation of the interlinkages is an attempt to systematise the relationships between ethics and sustainable development, which have already been enhanced in the literature but not analytically ordered by interconnecting separate variables of separate functions.

The interlinkages constitute the referential tools for a more explicit determination of ethically driven multidimensional goals of sustainable development.

\subsubsection{The interlinkages}

A general description of Table 3 is given, accounting for the interlinkages of each of the six macrovariables in Table 1.

- Improving competitiveness vs. governance vs. ethics - The economic dimension of sustainability is mainly expressed today through the variable of competitiveness. One main limitation of the concept of competitiveness, as such, is the scarce level of ethical notion embedded in the concept itself. The operability of the competitiveness is often interpreted to support economic benefit or power of small groups or individuals against others. Does this kind of interpretation align with the perspectives of sustainability? The new interlinkages establish a different concept of competitiveness, seeking both material and non-material profit and involving a high level of diversity (Table 3). The interlinkage orients the concept of competitiveness towards production and consumption mechanisms, based on systemic logic and reflexivity and material and non-material prosperity, repositioning citizens as manipulators of production and consumptions needs. 


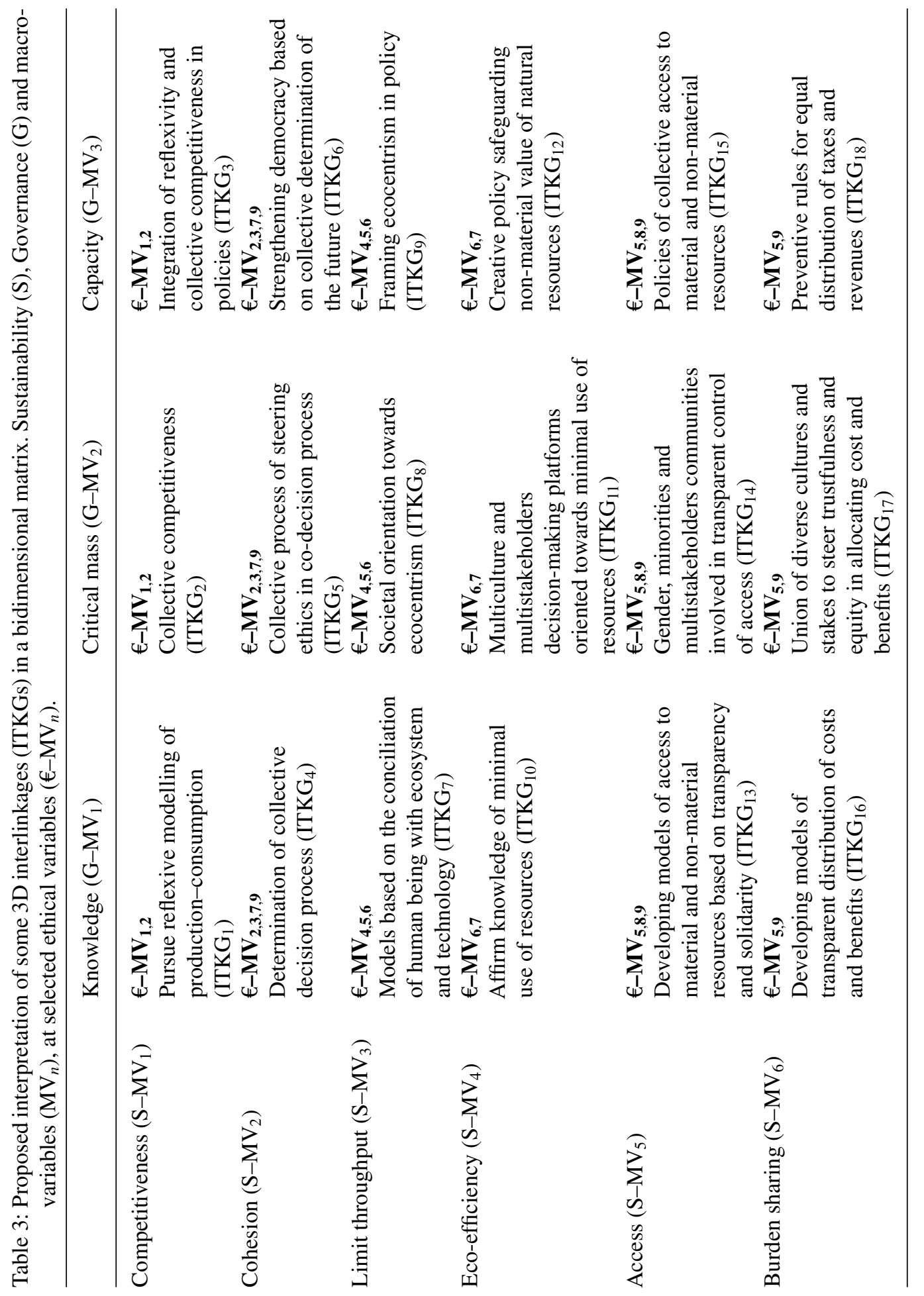


- Safeguard cohesion vs. governance vs. ethics - The opportunity of exploring the concept of cohesion in the new interlinkages enables a more explicit characterisation of paths and targets of the social dimension. Interlinking the social dimension with the knowledge and capacity variables and ethics places the emphasis on the concept of cohesion, built on the societal access to knowledge, to enhance the collective determination of the co-decision process or democratic process oriented towards the most relevant ethical principles (fraternity, peace, ecological enrichment, living in community, etc.). The link with the variable, critical mass, orients the formulation of cohesion based on diversities of people and knowledge in decision rights, prioritising the democratic process at the centre of a broad acceptance and determination of ethical values. The interlinkage gives the opportunity to further orient the concept of democracy, enhancing democracy not only on the base of the co-decision process, but also on the collective future determination of a democratic system centred on sustainable environmental, social and economic dimensions and ethics.

- Limit throughput vs. governance vs. ethics - The imperative of 'limit throughput' [14, 23] resembles the environmental imperative of sustainability, although it is not referenced by explicit governance and ethical variables. The regulation of material and spiritual conciliation of human being in ecosystem science-technology and the building of relevant knowledge channelled in the principle of dynamic ecosystem equilibrium and integrity are established by the new interlinkage. The new interlinkage orients the simple concept of environmental care towards the ethics of ecological enrichment shared on the base of transdisciplinary and trans-sector diversities of stakes, citizens and cultures, and tutoring with the support of creative policies.

- Eco-efficiency vs. governance vs. ethics - The variable 'eco-efficiency', established by the connection between the economic and environmental dimensions of sustainability [14], conceptually addresses accountability between the protection of natural resources (based on the concept of 'limit throughput') and the economic development. But the main limitation in conceptualising the interlinkage 'eco-efficiency' is the scarce level of ethical knowledge attached to the concept of eco-efficiency. The main question is: how should eco-efficiency be ethically pursued and on what ethical perspectives? Eco-efficiency as such does not explicitly express the orientation towards the minimisation of the use of resources in absolute terms. Eco-efficiency must leave the inspiration of traditional production-consumption models and valorise also the intrinsic non-material value of resource. The knowledge and capacity to develop ecosystem resources in terms of natural intrinsic value and minimal use forms the basis for the interlinkage between eco-efficiency, governance and ethical variables like ecological enrichment and ecological responsibility.

- Access vs. governance vs. ethics - The new interlinkage enhances individual and societal sanitation and happiness derived by access to resources that considers both material and spiritual satisfaction in the different sectors of use.

- Burden sharing vs. governance vs. ethics - The ethical value of solidarity and trustfulness in citizenship is embedded in the interlinkage. The interlinkage establishes the concept of strengthening transparency in cost and benefit repartition in the frame of a high rate of diversity in cultural and economic stakes and environmental needs. It also aims at building forms of regulation to ensure solidarity and justice in the distribution of costs and benefits due to use and manipulation of resources.

The set of new interlinkages can be used in multistakeholder public discourse to orient goal deliberation towards sustainable development. The interlinkages are the new multidimensional criteria in the process of goal prioritisation, while covering the balance of a broad sphere of elements of sustainable development. 


\section{THE CASE OF THE WASAMED PLATFORM}

Water SAving in MEDiterranean Agriculture (WASAMED) is a regional multistakeholder discussion platform formed in 2003 by the 5th RTD Framework Programme of the EC (http://wasamed.iamb.it). The network has 42 permanent members representing a majority of northern and southern Mediterranean countries [24]. The aim of the platform is to strengthen the dialogue among different actors and deliberate a common frame of goals and targets that respond to the need of harmonising agricultural water management within the global context of sustainable development in the Mediterranean. Indeed, the discourse on sustainable development in the Mediterranean started earlier with the Barcelona Convention in 1995, founded on the principles of north-south cohesion, cooperation and regional prosperity, and further strengthened in the 10th Meeting of the Mediterranean Commission on Sustainable Development [25].

\subsection{The background}

Building a communication platform on water saving in Mediterranean agriculture has been inspired by the fact that a sustainable management of natural waters in the region is possible with an effective water saving strategy. It is largely acknowledged that, among others, sustainable use of agricultural waters and related emissions is the basis for repositioning agriculture as an important socioeconomic and cultural asset of the region than it is today [26]. In the Mediterranean, natural water supply is unevenly distributed in space and time, while demographic growth exerts enormous pressure on water ecosystems. The main consumer of natural waters is agriculture, which uses up to $80 \%$ of this resource [27]. In the Mediterranean, the sustainable management of water in agriculture becomes vital to relieve the pressure on water withdrawal and develop local, national and transboundary environmental strategies. A sustainable management of agricultural water would than give a better impetus to integrate more explicitly the environmental imperatives in national and international agricultural policies [28], like for example the European Common Agricultural Policy (CAP). A sustainable management of agricultural waters needs to be supported by an effective water saving strategy focusing on different aspects and options: irrigation system performance, soil-plant related water use efficiency, water productivity and reuse of unconventional waters [29]. Also, an effective participatory irrigation management forms the basis for the successful implementation of the above water saving options. Sustainability, governance and cultural values, together with a certain degree of ethics, must be well embedded in local and regional strategies and be the common targets of the different actors to enable sustainable water management in agriculture. However, in the past, the development of agricultural water management has involved mainly sector actors. This has valorised more the intrinsic stakes of the agricultural sector, addressing the exclusive material benefits of farmers or other sector stakeholders and disregarding the dialogue with the society, the repositioning in the paths of global integration and strategies of reallocation of saved water volumes for other users and purposes. In other words, agricultural water management needs to seek perfect integration in the concept of societal or system competitiveness, while the future of agriculture, worldwide, is uncertain due to the emerging conflict with urbanisation, industry and tourism [26]. In the last two decades, we have assisted in the gradual exacerbation of the conflict between agricultural water users and the society, regarding the quality of the ecosystem. Agricultural water saving management has become more a tool to safeguard farm productivity and income rather than a tool for combating depletion of natural water yields, and improves societal access to, and use of, a cleaner ecosystem. 


\subsection{The process}

The activities of the WASAMED network started in January 2003. A series of international workshops have been conducted to strengthen regional dialogue and build shared knowledge on agricultural water saving and water management in the Mediterranean. Five workshops have been conducted in Sanliurfa (Turkey), Amman (Jordan), Hammamet (Tunisia), Cairo (Egypt) and Malta. Each workshop had more than 60 participants to bridge different positions and build a shared frame of goals for sustainable water management in agriculture. The participants included scientists from different disciplines, stakeholders such as decision makers, associations of farmers, NGOs representing the civil society, water boards and international organisations having a stake in the sector and the region [24]. Each workshop had an intense agenda consisting of key contributions from well-reputed scientists or experts to animate the discussion in the plenary sessions and working groups. In each workshop, members were split into homogeneous working groups with expertises in socioeconomic, environmental, governance and technical aspects. The outputs of the plenary sessions and working groups have been the basis of the three-step application of the conceptual method described before.

The first step was to collect ideas for the future by asking participants about their ideas for sustainable water management. This was done in the first workshop, which broadly focused on the social, socioeconomic and cultural aspects of water management. The participants were asked to formulate their ideas on: (1) how to ensure water to end-users, (2) how to integrate the local culture into the water management process, (3) how to improve participation and co-decision in water management in agriculture, (4) how to develop paths of modernisation, (5) how to improve the local empowerment and the allocation of water. The outputs were collected to compose the leitbild $[14,30]$ of the WASAMED platform (Table 4).

The second step was the collective deliberation in the four successive workshops, where participants expanded the preliminary leitbild to the form of 1D or 2D goals respectively, at each corner and each bidimensional interlinkage of the triangles of sustainability (Fig. 5) and governance (Fig. 6). The deliberation process has produced a high number of outputs, which have been organised and systematised with the use of the sustainability and governance triangles. The members of the working groups prioritise goals of sustainability and governance and compare them with the macro-variables given by the conceptual model. Indeed, the deliberated goals constitute the mean for the operability of the macro-variables given in the theoretical framework described before.

The third step was the definition of the multidimensional goals based on the frame of conceptual multidimensional interlinkages given in Table 3 . These goals are deduced by interpreting the significance of combining simultaneously the separate goals of sustainability and governance at the

Table 4: An example of ideas collected in the first step and forming the liebild of the platform.

Social concern

Equity in water allocation, knowledge and education for water end-users, transparency in decision making, minimum water storage by farmers, agricultural water market policy, dialogue with policy makers

Environmental concern Water quality, knowledge of good ecosystem practices, effective operation and maintenance of water systems

Economic concern Water productivity

Governance Water rights, involvement of gender in water management, transparency in information 
selected ethical variables given by the model. In practical terms, once sustainability and governance goals (Figs. 5 and 6) were deliberated by the groups, a very small group of experts derived the most plausible multidimensional goals (Table 5). This work shows that it is possible to translate the frame of goals of Figs. 5 and 6 into a more comprehensive frame of multidimensional goals (thus the new S-G-€ interlinkages of WASAMED) oriented with explicit values of ethics. Indicators were attached to each multidimensional goal. The more focused and localised the multidimensional goal, the easier it was to attach an indicator. Indicators are preferably simple, having mainly ordinal or nominal characters, easy to understand and not necessarily based on highly technical existing data, given the possibility that data collection of regional and local institutions are often limited. Not all the possible combinations of $\mathrm{S}$ and $\mathrm{G}$ goals deliberated in step 2 have multidimensional goals defined or combinations worked out with a perfect equal weightage given to all of them. However, it is obvious

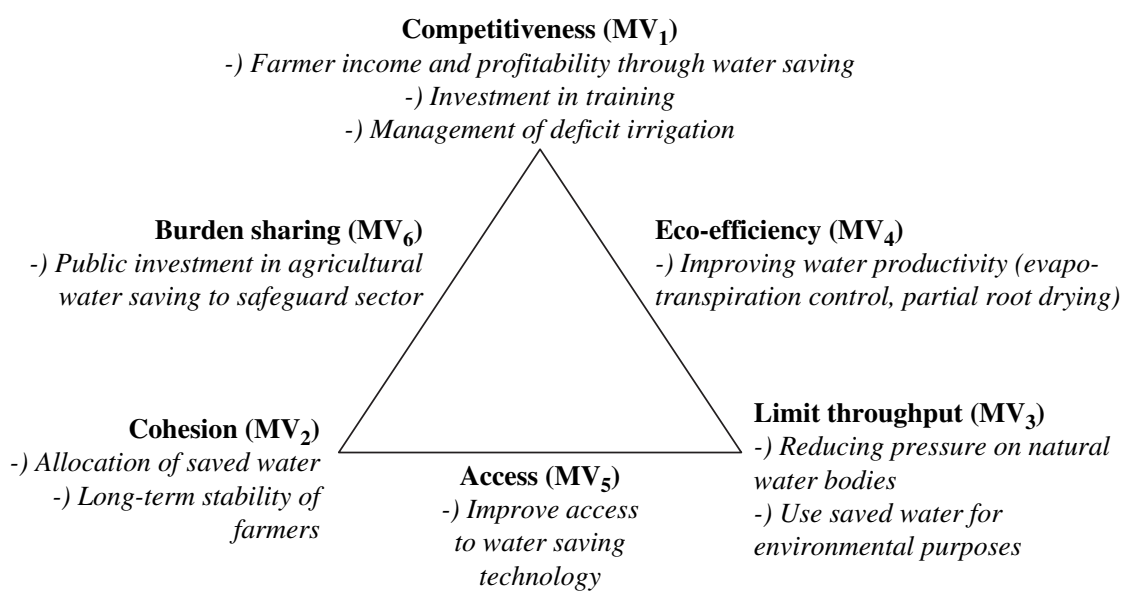

Figure 5: Some examples of sustainability goals deliberated by the stakeholder groups.

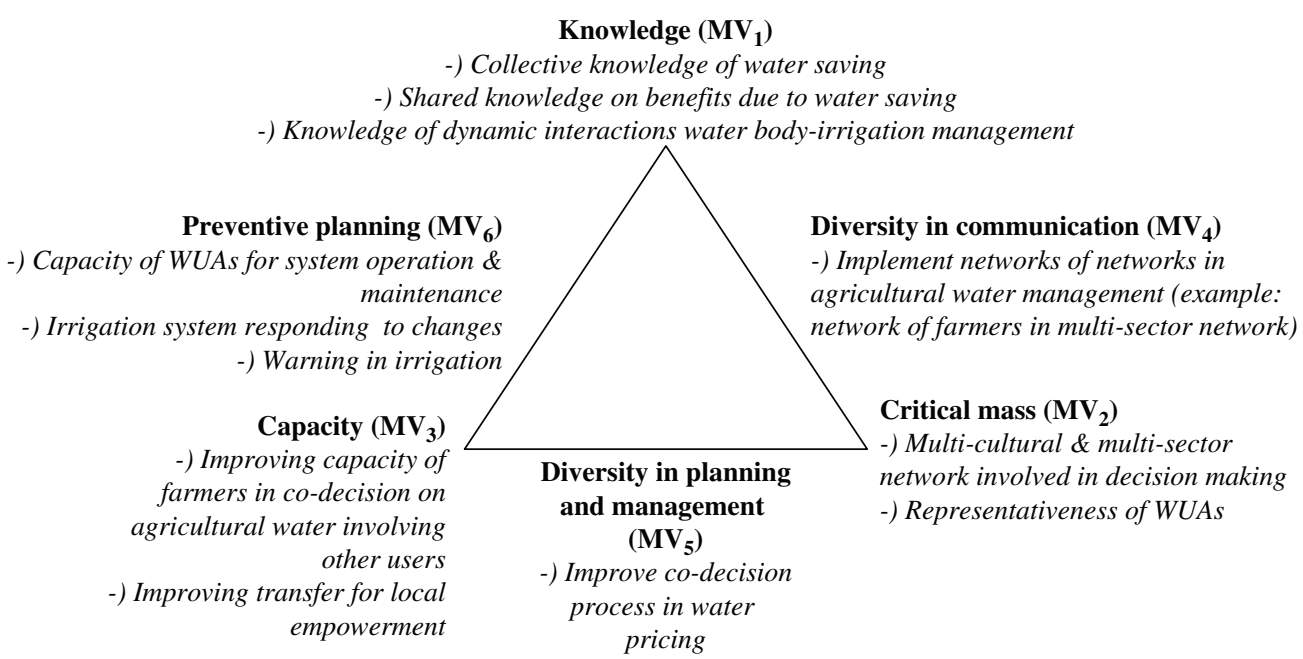

Figure 6: Some examples of governance goals deliberated by the stakeholder groups. 


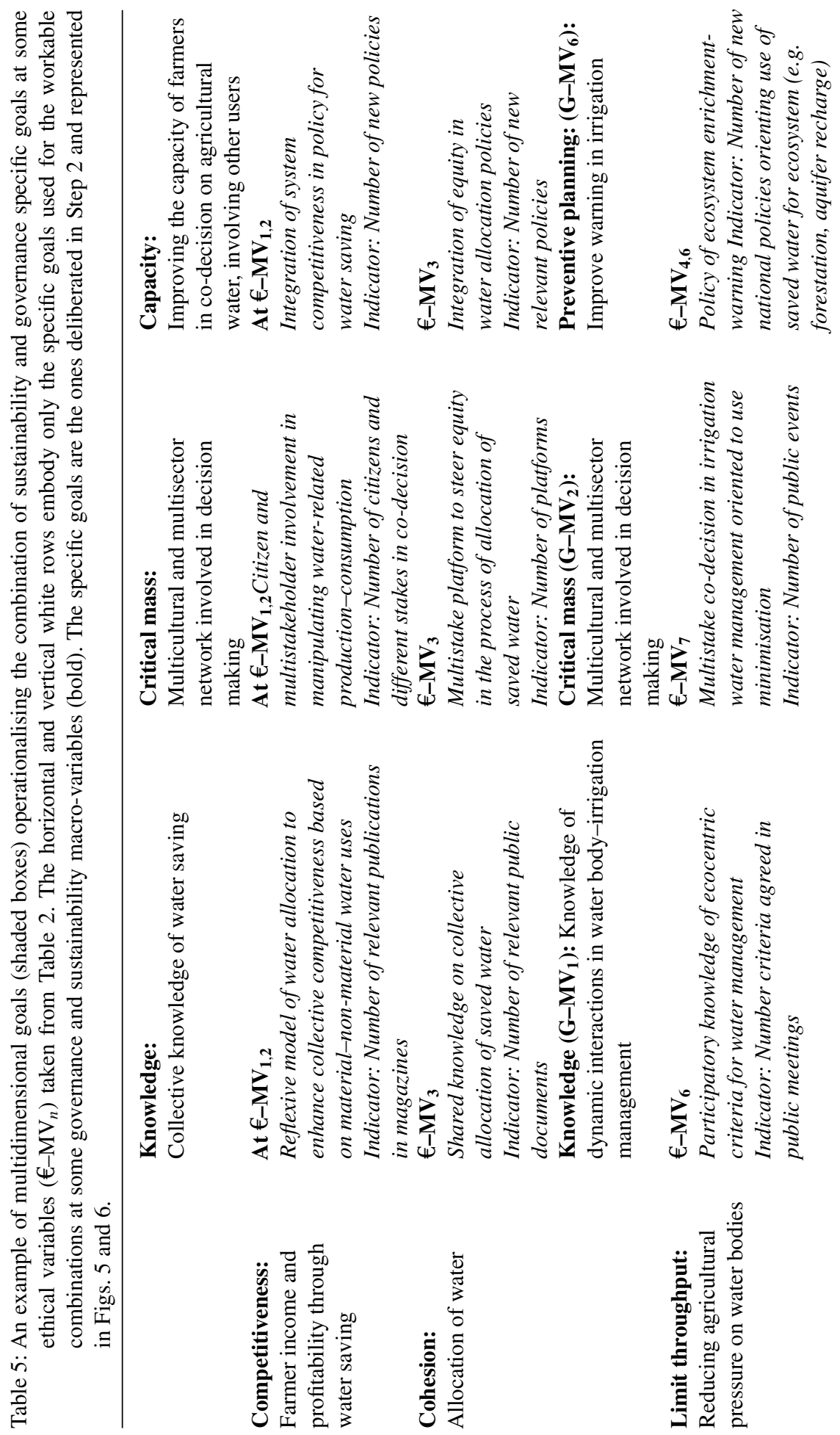




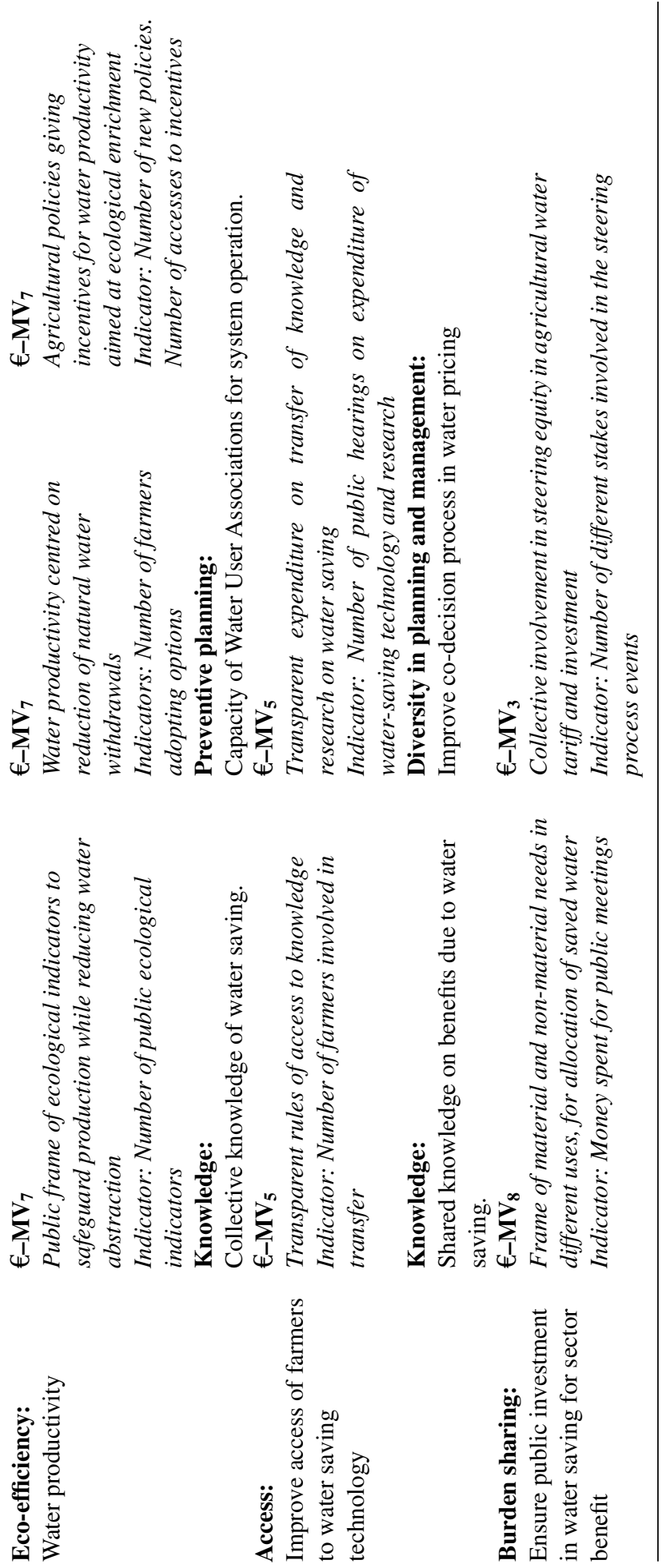


that new multidimensional goals are not all directed to one or the other function of sustainability, governance or ethics but are distributed with a rather good balance. Moreover, because any other platform discourse different from WASAMED would deliberate other ideas, we should expect that the multidimensional goals must be different as well, but as a starting point, they may be deliberated and combined in the form that most suits the conceptual purpose of the conceptual model. Thus, the new multidimensional goals defined in Step 3 can be a useful example, but not a blueprint for other cases. The next steps in the process would be the visualisation of the entire frame of multidimensional goals in the national and local agendas on agricultural water management and the enhancement of their explicit inclusion in programmes and projects.

\subsection{Discussion}

The modern debate on water use and sustainable development is based on the assumption that managing natural resources is an ethical imperative, but rarely questions the basis for this viewpoint [31]. The application of the model to WASAMED platform gives the opportunity to define multidimensional goals for agricultural water management. Consequently, it helps to identify some bases that could underlie such an assumption, seeking to locate ethical values in the water agendas beyond anthropocentric utilitarianism. The multidimensional goals derived by the application of the model suggest a clear basis for this type of debate aiming at resolving issues and conflicts that arise in the management of water between: present and future, human and non-human uses, competing human users, material and non-material uses, reflexive and non-reflexive ways to use water for socioeconomic growth, personal and collective competitiveness and equity and non-equity in access to resources. It gives to agricultural water management other perspectives than simply looking after sector productivity and competitiveness. It brings about a more systematic integration of the guiding concept of ethics into the concept of water sustainability. The systematisation of outcomes deliberated in the process gives the opportunity to identify more clear perspectives of sustainable agricultural water management beyond the sector interest, abandoning the concept of water use in the Mediterranean as property of the agricultural sector and leaving the traditional business-as-usual and non-systemic socioeconomic approach $[26,32,33]$. These perspectives are embedded and organised in explicit and clear dynamic of interlinkages among sustainability, governance and ethical principles (Table 5): equity in access and use, collective responsibility and competitiveness, diversity of culture and material/non-material stakes in co-decision and strategy, creative policies, water resources enrichment and transparency and trust in building shared knowledge.

Further use of the multidimensional goals, together with an improvement of their conceptual meaning in the water sector, will be a good starting base to formulate useful criteria for the integration of agriculture and related water management at a more global level in view of a more multifunctional agriculture, as indicated also by other authors [34].

\section{CONCLUSIONS}

The innovative character of the proposed approach is based on both conceptual and methodological features. The integration of explicit macro-variables of $S, G$ and $€$ into a multilevel set of $S-G-€$ interlinkages helps to achieve a holistic, systematised and explicit representation of multidimensional paths of sustainable development. The new interlinkages are explored by a heuristic matrix that structures them for the determination of goals of sustainable development based on the simultaneous balance among the socioeconomic-environmental dimensions of sustainability and factors of governance and ethics. The $\mathrm{S}-\mathrm{G}-€$ field helps to comprehensively frame sustainable development in goal deliberation, which can be useful to develop early-stage project logical framework, programmes 
or sector strategies, improving the transferability of interpretation criteria to different conditions. In respect to other existing conceptual methods, the $\mathrm{S}-\mathrm{G}-€$ field is a more complex device - but more comprehensive - due to attempts to explore the complexity of a 3D system. The model provides the means for the multidimensional structuring and evaluation of goals based on the explicit influence of ethics in the interpretation of sustainability and governance criteria. The introduction of ethics, as a separate domain, although complementary to sustainability and governance, emerges as a need to avoid fragmentation of visions of sustainability and consequent risk of shifting away from the genuine common objectives of sustainable development. Attention must be given to the kind of approach and not to the type and number of variables or ethical principles used. What matters is the option provided by the heuristic framework of leaving the design and assessment of sustainable development based on a reductive and implicit approach. It might not be sufficient in the future to design and evaluate sustainable development with the exclusivity of traditional sustainability criteria. In conclusion, the paper intends to stimulate the scientific community as well as project/programme makers, evaluators and practitioners to consider a different way of framing goals of sustainable development.

\section{REFERENCES}

[1] World Commission on Environment and Development, Our Common Future, Oxford University Press: London, 1987.

[2] Sampford, C., Environmental governance for biodiversity. Environmental Science \& Policy, 5, pp. 79-90, 2002.

[3] De Paula, G.O. \& Cavalcanti, R.N., Ethics: essence for sustainability. Journal of Cleaner Production, 8, pp. 109-117, 2000.

[4] Robinson, J., Squaring the circle ? Some thoughts on the idea of sustainable development. Ecological Economics, 48, pp. 239-384, 2004.

[5] Meppen, T. \& Gill, R., Planning for sustainability as a learning concept. Ecological Economics, 26, pp. 121-137, 1998 .

[6] Jickling, B., A future for sustainability? Water, Air and Soil Pollution, 123, pp. 467-476, 2000.

[7] Commission of the European Communities, Working together for growth and jobs - A new start of the Lisbon Process - SEC, pp. 192 and 193, 2005.

[8] European Commission, The 6th RTD Framework Programme, www.cordis.lu

[9] Spangenberg, J.H., Omann, I. \& Hinterberger, F., Minimum benchmarks and scenarios for employment and the environment. Ecological Economics, 42 pp. 429-443, 2002.

[10] Spangenberg, J.H., Pfahl, S., Deller, K., Towards indicators for institutional sustainability: lessons from an analysis of Agenda 21, Ecological Indicators, 2 pp. 61-77, 2002.

[11] Serageldin, I., Sustainability and the Wealth of Nations, First Steps in an Ongoing Journey, World Bank: Washington, DC, 1996.

[12] Funtowicz, S., Ravets, J. \& O'Connors, M., Challenges in the use of science for sustainable development, Int. J. Sustain. Dev., 1, 1998.

[13] Rosner, W.J., Mental models for sustainability. Journal of Cleaner Production, 3, pp. 107-121, 1995.

[14] Valentin, A. \& Spangenberg, J.H., A guide to community sustainability indicators. Environmental Impact Assessment Review, 20, pp. 381-392, 2000.

[15] Kant, I., Kritik der praktichen, Vernunft, Köninsberg, 1788.

[16] United Nations, Indicators of Sustainable Development, Framework and Methodologies. United Nations: New York, 1996.

[17] Rodhes, R.A.W., The new governance: governing without government. Political Studies, XLIV, pp. 652-667, 1996. 
[18] European Commission, The European Governance, COM 428, Commission of European Communities: Bruxelles, 2001.

[19] Chaskin, R.J., Building Community Capacity, Aldine De Gruyter: New York, 2001.

[20] Balakrishnan, U., Duvall, T. \& Primeaux, P., Rewriting the bases of capitalism: reflexive modernity and ecological sustainability as the foundations of a new normative framework. Journal of Business Ethics, 47, pp. 299-314, 2003.

[21] Collins, D., Virtuous individuals, organisations and political economy: a new age theological alternative to capitalism. Journal of Business Ethics, 26, pp. 319-340, 2000.

[22] Weil, P., The new ethics, Editora Rosa dos Tempos, 1998.

[23] Spangenberg, J.H., Environmental space and the prism of sustainability: frameworks for indicators measuring sustainable development. Ecological Indicators, 2, pp. 295-309, 2000.

[24] WASAMED (Water Saving in Mediterranean Agriculture), http://iamb.wasamed.it

[25] United Nations, Report of the tenth Meeting of the Mediterranean Commission on sustainable development (MCSD), UNEP (DEC)/MED WG.277/4, Athens, June 2005.

[26] Safley N., How traditional agriculture is approaching sustainability, Biomass and Energy, 14, pp. 329-332, 1998.

[27] Global Water Partnership Forum, Regional action, Mediterranean, http://www.gwpforum.org

[28] Zalidis, G.C., Tsiafouli, M.A., Takavakoglou, V., Bilas, G. \& Misopolinos, N., Selecting agrienvironmental indicators to facilitate monitoring and assessment of EU agri-environmental measures effectiveness. Journal of Environmental Management, 70, pp. 315-321, 2004.

[29] Bogliotti, C., The Mediterranean perspective. Proceedings of the International Conference of the European Commission, Research Directorate-General: European Water Scenarios: From the Mediterranean Sea to Central Asia, Brussels, 30 June-1 July 2003.

[30] Dierkes M., Lietbild und Technik, Edition Sigma: Berlin, 1992.

[31] Armstrong, A., Ethical issues in water use and sustainability. AREA, 38(1), pp. 9-15, 2006.

[32] Hansen J.W., Is agricultural sustainability a useful concept? Agricultural Systems, 50, pp. 117-143, 1996.

[33] Van der Helm, R., Challenging future studies to enhance EU's particpatory river basin management. Physics and Chemistry of the Earth, 28, pp. 563-570, 2003.

[34] Chrispeels, M.J. \& Mandoli D.F., Agricultural ethics. Plant Physiology, 132, pp. 4-9, 2003. 